\title{
Noise Reduction based on Multiple Copies Color Image Noise Estimation
}

\author{
Napa Sae-Bae ${ }^{1}$ and Somkait Udomhunsakul ${ }^{2}$ \\ ${ }^{1}$ Computer Science Department \\ ${ }^{2}$ Engineering and Architecture Department, Rajamangala University of Technology \\ Suvarnabhumi, Nonthaburi, Thailand \\ benapa@gmail.com, sudomhun@hotmail.com
}

\begin{abstract}
Nowadays, transmission in the form of color digital images is becoming a major method of communication. However, color digital images are often degraded by noise generated by sensors and during transmission channels. The aim of the noise reduction is to remove noise while keeping the important image features as much as possible. In this paper, a novel method to remove additive noise from color digital images, based on multiple copies of color image noise estimation, is proposed. The proposed method, noise variance in each band: red, green and blue, is estimated separately from noisy color images using the information of an original color image from another copy of noisy color images. This proposed noise estimation could be used in conjunction with the state-of-the-art denoising techniques to improve the quality of reconstructed images. Experiments to evaluate the performance and characteristic of the proposed noise estimation technique are performed on ten image datasets in various contents and wide range of noise variances. The results show that the proposed approach can be used to reconstruct color noisy images with better quality, in terms of PSNR and visual perception compared to an original wavelet shrinkage denoising technique particularly for the images with many high frequency components.
\end{abstract}

Keyword: Denoising, Noise reduction, Gaussian additive noise, Multiple noisy images, Color images

\section{Introduction}

Removing noise from noisy images is an essential research in the field of digital image processing. Indeed, color digital images are often degraded due to sensors imperfection and transmission channels defects, where noise deteriorates the quality of almost every acquired color digital images [1]. In general, when filtering random noise from a noisy image, there are two main issues of noise reduction that need to be considered, which are how much noise had been removed and how well edges are preserved. Hence, to get rid of noise without distorting information in the image is a challenging task. Traditionally, there are simple techniques for noise suppression such as Moving average filter and Gaussian filter. These simple techniques can effectively suppress noise but, being merely a low pass filter, they fail to preserve many useful detail [2]. This leads to search for noise filtering technique alternatives.

In the past decades, Wavelet Transform has been used as a powerful technique to recover signal from noisy data. This method is commonly referred to wavelet shrinkage techniques. In 1995, a soft thresholding for denoising in 1-D signal was proposed [3]. Later, in 2000, S. Chang, B. Yu and M. Vetterli introduced an adaptive wavelet thresholding for image denoising and compression [4]. Specifically, they proposed a new shringkage method, BayesShrink, which outperformed Donoho and Johnstone's Sureshrink. In addition, number of adaptive waveletbased image denoising methods based on thresholding and some Wavelet based shrinkage methods, were proposed and studied [5]-[7]. However, Wavelet denoising methods has two main drawbacks, which are the choice of the threshold and the specific distributions of the signal and noise may not be well matched at different scales [8]. Other alternatives, smooth region's mean deviation-based denoising methods by using two different filtering window sizes to achieve an optimum reservation of the image fine details and edges are also proposed [9]. Specifically, the utilization of the mean deviation in the determination of the threshold value contributes to a more

Received: January $31^{\text {th }}, 2018$. Accepted: July $27^{\text {th }}, 2019$

DOI: $10.15676 /$ ijeei.2019.11.3.5 
accurate division of smoothing and non-smoothing regions. In 2011, there were researchers introduced a method for removing noise while preserving the image fine details and edges in blind condition, based on Wiener filter and a constructed edge map [10].

Despite the richness of work on the noise suppression of gray scale images, color image noise reduction has received considerably much more attention and getting extremely popular nowadays. Therefore, color image noise reduction is also an essential task in digital image processing systems. Recently, an advanced color image denoising scheme called multichannel nonlocal means fusion (MNLF) and multi-channel circular spatial filter (MCSF) were developed for color image denoising [11]-[12]. Another denoising algorithm, a new denoising method, based on the minimum cut algorithm, to exploit both the interscale and intrascale correlations of wavelet coefficients was presented [13]. All these are the techniques to reduce additive noise from a single image.

In some applications, a single image could be corrupted by noise for multiple times. Consequently, researchers have proposed noise reduction techniques that can utilize information from those multiple noisy copies of the same image source more effectively and resulting in the recovered image with better quality as compared to the traditional approaches. In 2000, S. G. Chang et al have studied the impact of ordering between thresholding and fusing steps towards the enhancement of image quality when multiple copies of images are available [14]. More recently, Youssef et al have proposed the hierarchical multistage nonlinear filtering techniques to reduce noise on medical images [15].

In our previous work, we have proposed another alternative, the noise estimation technique for multiple noisy gray scale image copies [16]. One application of this proposed technique was to be used in conjunction with another image noise reduction technique [17]-[18] to improve the quality of the recovered image in such scenario. In addition, the proposed technique can be applied to color images denoising. In fact, the color image processing significantly differs from monochrome image processing because of the redundancy and the complementary information within the color bands. The processing is much more complicated and hence the need to extract and exchange information from and among all bands. However, in general thought, these seem to be an agreement we can process each of the three monochrome images separately and combine the results, which imply that it is sufficient to denoise brightness only and, in a sense, treat a color image like an achromatic one [19]. The proposed method, noise variance in each band: Red, Green and Blue, is estimated separately from noisy color images using the information of an original color image from another copy of noisy color images when multiple noisy color image copies are available.

In this paper, we focus on color image denoising method from multiple copies of noisy images. We extend the previously proposed method to estimate noise variance on grey-scale images when multiple copies of images are available. Specifically, a noise variance of each noisy image is estimated in each band: red, green and blue, individually. Then, for each color band, this estimated noise variance is used in conjunction with wavelet shrinkage denoising technique [17] to reconstruct noise-free image. In addition, we perform extensive evaluation of the quality of the images recovered by the proposed denoising technique using objective and subjective measurements. Finally, we provide insights on the image characteristic that would greatly benefit from our proposed technique. Specifically, the proposed technique utilizes information from another noisy image copy in each band to search for the optimal noise variance based on the assumption that these image copies are originated from a single source and that they are corrupted by Additive white Gaussian noise (AWGN).

The paper is organized as follows. The next section presents a brief background of noise reduction and spatial frequency measure. In section III, the methodology of proposed color image denoising method is provided. Next, some experimental results to quantitative and qualitative the effectiveness of the proposed method as well as comparison with the performance of another existing method are expressed in section IV. Finally, concluding remarks are given in section V. 


\section{Background}

In this section, background related to our proposed noise reduction model is provided.

\section{A. Additive Gaussian noise}

Generally, image noise can be divided into two types: additive and multiplicative noise. AWGN is an additive noise signal that each sample is drawn from Gaussian distribution with zero mean and variance $\sigma$. Specifically, the probability density function, denoted by $f(x)$, of Gaussian or normal distribution with zero mean and variance is defined as:

$$
f(x)=\frac{1}{\sqrt{2 \sigma^{2} \pi}} e^{-\frac{x^{2}}{2 \sigma^{2}}}
$$

For a noisy color $N$ of a true color image $C$ is formulated as

$$
N=C+n
$$

where $n$ is the additive independent and identically distributed Guassian noise having zero mean and variance $\sigma$.

\section{B. Wavelet based denoising technique}

The theoretical formalization of filtering additive Gaussian noise (of zero-mean and standard deviation) via thresholding wavelet coefficients was pioneered by Donoho and Johnstone [4]. A wavelet coefficient is compared to a given threshold and is set to zero if its magnitude is less than the threshold; otherwise, it is kept or modified (depending on the thresholding rule) [5]. In this paper, the wavelet denoising method based on bivariate shrinkage functions, proposed by Selesnick et al., is adopted as an algorithm to reduce noise on a single noisy image [17]. The implementation used in this work is available to download [18]. In addition, it is used in conjunction with our proposed noise estimation to get the reconstructed color images.

Spatial frequency measure

The spatial frequency measure (SFM) indicates the overall activity level in an image [20].

$$
\begin{aligned}
& S F M=\sqrt{R^{2}+C^{2}} \\
& R=\sqrt{\frac{1}{M N} \sum_{m=1}^{M} \sum_{n=2}^{N}(x(m, n)-x(m, n-1))^{2}} \\
& C=\sqrt{\frac{1}{M N} \sum_{m=1}^{M} \sum_{n=2}^{N}(x(m, n)-x(m-1, n))^{2}}
\end{aligned}
$$

where $R$ is row frequency, $C$ is column frequency, $x(m, n)$ denotes the samples of image, $M$ and $N$ are number of pixels in row and column directions, respectively. The large value of SFM means that image contain component in high frequency area.

\section{Methodology}

In this section, the proposed image denoising method is subdivided into three steps, presented in figure 1.

Step 1: Two noisy color images are separately extracted into three subbands, Red Green and Blue.

Step 2: Noise in each Red $\left(R_{1} R_{2}\right)$, Green $\left(G_{1} G_{2}\right)$ and Blue $\left(B_{1} B_{2}\right)$ band, is separately estimated, fused and then denoised.

Step 3: The results from step 2 in each band, $\mathrm{R} \mathrm{G}$ and B, are combined to get a reconstructed image.

Specifically, in step 2, the proposed noise estimation technique is developed and used in conjunction with the well-known wavelet denoising algorithm [17]. Moreover, image fusion need to be performed before denoising process in order to get the reconstructed image. Details for each process are described as follows. 


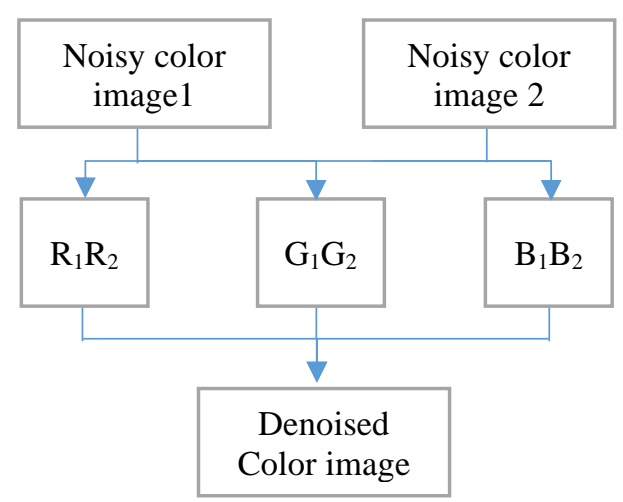

Figure 1. Block diagram for proposed method

\section{A. Noise estimation}

For denoising algorithm, the threshold is typically computed as a function of noise variance. Given a single noisy image copy, this noise variance is often estimated using median absolute deviation (MAD) technique, the most well-known thresholding methods (VisuShrink) [4]. In this work, we argue that when multiplecopies of noisy images become available, those noisy images can coordinately be used to estimate the level of noise variance more accurately than the traditional method, namely the MAD estimation. Specifically, noise variance can be search for by optimizing the mean square error (MSE) between the denoised image and another noisy image. For simplicity, this paper only considers the case when two copies of noisy images are available. Let $R$ be the red band of original color image and R1 and R2 be the first and the second copy of noisy color images corrupted by Additive white Gaussian noise (AWGN) with noise variance $\sigma 1$ and $\sigma 2$ accordingly. Then, $R_{1}[i, j]$ and $R_{2}[i, j]$ can be formulated as [17]:

$$
\begin{aligned}
& R_{1}[i, j]=R[i, j]+N\left(0, \sigma_{1}\right) \\
& R_{2}[i, j]=R[i, j]+N\left(0, \sigma_{2}\right)
\end{aligned}
$$

Let $R_{1}{ }^{\prime}\left(\sigma_{1}{ }^{\prime}\right)$ be the denoised image derived from $R_{1}$ when noise variance is estimated at $\sigma_{1}{ }^{\prime}$. The objective function is to search for the noise variance that minimizes the Mean Standard Error (MSE) between $R_{1}$ and $R_{2}$. That is:

$$
\sigma^{(1)}=\operatorname{argmin}_{\sigma_{1^{\prime}}} \mid\left\|R_{2}-R_{1}{ }^{\prime}\left(\sigma_{1}{ }^{\prime}\right)\right\|_{2}
$$

Similarly, the noise variance of $R_{2}$ can be estimated from:

$$
\sigma^{(2)}=\operatorname{argmin}_{\sigma_{2^{\prime}}}\left\|R_{1}-R_{2}{ }^{\prime}\left(\sigma_{2}{ }^{\prime}\right)\right\|_{2}
$$

\section{B. Image fusion technique}

When multiple noisy images are available, the recovered image is typically constructed by fusing those images using linear combination whether before or after denoising. If the fusion is performed before denoising, the fusion can be computed as the weighted average at pixel-wise level as follows [1].

Let $R_{i}$ be thedenoised version for each copy of the noisy image. Each pixel of the fused image is computed as:

$$
R_{f}[i, j]=\sum_{i=1}^{N}\left(w_{n} \times R_{i}[i, j]\right)
$$

where optimal weight, $\mathrm{w}_{\mathrm{n}}$, for each of the image copies is given by [15]:

$$
w_{n}=\frac{1}{\sigma_{i}^{2}} \times \frac{1}{\sum_{j=1}^{N} \frac{1}{\sigma_{j}^{2}}}
$$

where $\sigma_{i}$ is the noise variance for each copy of the noise image. Then the fused image can be denoised using the following noise variance estimation:

$$
\sigma^{f}=\frac{1}{\sqrt{\sum_{i=1}^{N} \frac{1}{\sigma_{j}{ }^{2}}}}
$$




\section{Objective image quality assessment}

In general, image quality evaluation can be classified into two methods, which are subjective measurement and objective measurement. For objective measurement, it is save time more than subjective quality measurement. The simplest and most widely used full-reference quality metric is the mean squared error (MSE), computed by averaging the squared intensity differences of distorted and reference image pixels, along with the related quantity of peak signal-to-noise ratio (PSNR). These are appealing because they are simple to calculate, have clear physical meanings, and are mathematically convenient in the context of optimization [21]. Therefore, PSNR is selected and used for this research work study.

For mean squared error (MSE), it can bedefined as:

$$
\left.M S E=\frac{1}{M N} \sum_{m=1}^{M} \sum_{n=1}^{N}\left(I[i, j]-I^{\prime}[i, j]\right)^{2}\right)
$$

where $M$ and $N$ are the width and height of the image. Also, $I[i, j]$ and $I^{\prime}[i, j]$ are the intensity of the reference image pixel and the recovered image pixel at the position $(i, j)$, respectively. Because many signals have a wide dynamic range, PSNR is usually expressed in terms of the logarithmicdecibel scale. It is most easily defined via the mean squared error (MSE) as:

$$
P S N R=10 \log \frac{\left(2^{d}-1\right)^{2}}{M S E}
$$

Here $d$ is the number of bits that are used to represent the intensity of pixel. When the pixels are represented using 8 bits per sample, $\left(2^{d}-1\right)$ is equivalent to 255 . That is, the larger the PSNR value is, the better performance the denoising method is.

For color peak signal-to-noise ratio $(P S N R c)$ [22], 8bits per pixel,

$$
P S N R_{C}=10 \log \frac{3 \times\left(2^{d}-1\right)^{2}}{M S E_{r}+M S E_{g}+M S E_{b}}
$$

where $M S E_{r}, M S E_{g}$ and $M S E_{b}$ are the mean squared error of the red, green and blue band between distorted and reference image pixels, respectively.

\section{Experimental results}

One main challenge in testing color image denoising method is how to decide which test images to use for the evaluations. The image content being viewed influences the perception of quality irrespective of technical parameters of the denoising method [20]. Ten test images $(256 \times 256,8$ bits/pixel) that have different spatial characteristics, are shown in Figure 2. (some of them are available to download at [26]). In addition, SFM are used to quantify the amount of information (details) for original color images (See Table I). It is seen that, original images house and baboon have the lowest SFM (17.08) and highest SFM (39.2). Baboon, hen, and cat images which correspond to a large value of SFM have a lot of details. Generally, larger value of SFM would correspond to an image that contains more high frequency components.

In order to generate colour noisy image, each test image are corrupted with additive (synthetic) Gaussian white noise having the same noise variance at 5, 8 and 10 in each red, green and blue band. These noisy images are then denoised by bivariate wavelet shrinkage using two noise variance estimation techniques: (1) the proposed method as well as (2) a robust median estimator using finest scale wavelet coefficients band [17]. For the sake of fair comparison, the reported performance of the original approach [17] is obtained by denoising the color image individually. Then, the reconstructed image is derived by fusing all denoised image with the algorithm described in the previous section. The PSNRc comparison between the two noise variance estimation techniques are reported in Table II, with the best results shown in bold. The results clearly demonstrate the effectiveness of the proposed noise estimation techniqueparticularly when the original color image having high SFM (cat, hen and baboon). That is, for these high SFM images, the proposed noise estimation technique results reconstructed images with much higher PSNRc compared to the original bivariate wavelet shrinkage denoising with a robust median estimator [17] (2.18 PSNRc improvement on average for the last three images, compared to -0.44 for the first 7 images). 
Napa Sae-Bae, et al.

Moreover, our proposed algorithm can be applied for noise reduction not only in a low noise density, but a high noise density as well. Next, four standard well-known color images (Pepper, Lena, Barbara and Barboon) are used as test images [25]-[26]. All color images are of 512×512, 8 bits/pixel. Again, these images are corrupted with additive

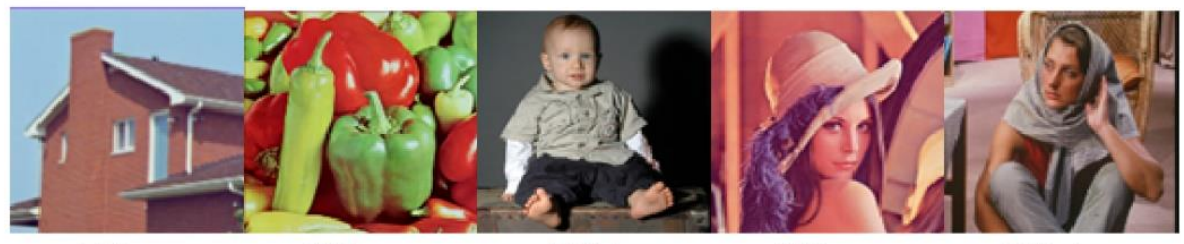

(a) House

(b) Pepper

(c) Baby

(d) Lenna

(e) Barbara

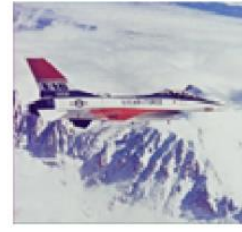

(f) Airplane

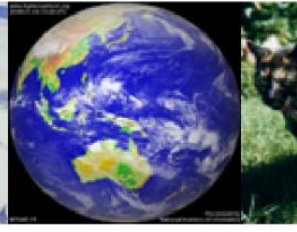

(g) Earth

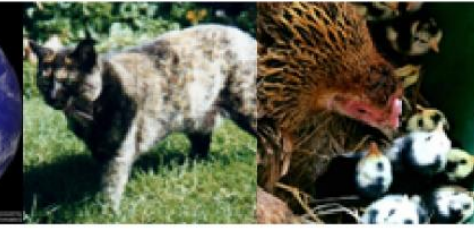

(h) Cat

(i) Hen

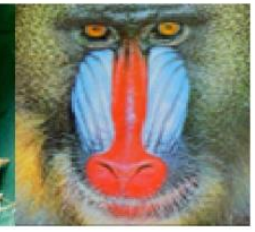

(j) Baboon

Figure 2. Ten original test images

Table 1. SFM values for ten test images

\begin{tabular}{|l|l|}
\hline Image name & SFM \\
\hline house & 17.08 \\
\hline pepper & 23.11 \\
\hline baby & 24.33 \\
\hline lena & 24.35 \\
\hline barbara & 25.24 \\
\hline airplane & 29.55 \\
\hline earth & 31.5 \\
\hline cat & 36.01 \\
\hline hen & 38.6 \\
\hline baboon & 39.2 \\
\hline
\end{tabular}

Table 2. PSNRc of the denoised images generated from two denoising algorithms

\begin{tabular}{|c|c|c|c|c|c|c|}
\hline \multirow{2}{*}{ Image name } & \multicolumn{2}{|c|}{ noise variance $=5$} & \multicolumn{2}{c|}{ noise variance $=8$} & \multicolumn{2}{c|}{ noise variance $=10$} \\
\cline { 2 - 7 } & \multicolumn{2}{|c|}{ PSNRc } & \multicolumn{2}{c|}{ PSNRc } & \multicolumn{2}{c|}{ PSNRc } \\
\cline { 2 - 7 } & Proposed & Ref [17] & Proposed & Ref [17] & Proposed & Ref [17] \\
\hline house & 38.45 & 38.667 & 35.627 & 36.157 & 34.581 & 35.032 \\
\hline peper & 37.577 & 38.368 & 35.116 & 35.728 & 34.152 & 34.454 \\
\hline baby & 39.024 & 39.698 & 36.119 & 36.519 & 34.894 & 34.979 \\
\hline lena & 37.73 & 38.644 & 35.804 & 35.967 & 34.081 & 34.658 \\
\hline berberra & 37.924 & 37.932 & 34.695 & 34.956 & 33.856 & 33.55 \\
\hline ariplane & 37.177 & 38.818 & 34.964 & 35.873 & 33.634 & 34.477 \\
\hline earth & 37.242 & 37.552 & 34.275 & 34.274 & 32.791 & 32.74 \\
\hline cat & 37.243 & 33.945 & 33.193 & 31.851 & 31.91 & 30.699 \\
\hline hen & 37.8 & 36.562 & 34.27 & 33.534 & 32.545 & 32.068 \\
\hline baboon & 37.148 & 31.654 & 33.276 & 30.126 & 31.96 & 29.207 \\
\hline
\end{tabular}


(synthetic) Gaussian white noise having the same noise variance at 10, 15 and 20 in each red, green and blue band. In addition to PSNRc, IFS index is used to measure the image quality for this experiment. IFS or an independent feature similarity is an index for full-reference color image quality assessment [23]-[24]. The computation of IFS consists of two components: feature component and luminance component. The feature component measures the structure and texture differences between reference and distorted images, while the luminance component evaluates brightness distortions. Compared with other image quality assessments, IFS performs very well on additive white Gaussian noise (AWGN). The PSNRc and IFS comparison results are tabulated in Table III and IV, with the best results shown in bold. In addition, Figure 5 shows the PSNR versus noise variance plotting results. From the results, it clearly demonstrates the effectiveness of the proposed noise estimation technique had similar or slightly closed but performs better than well-known wavelet based denoising [17], bivariate shrinkage functions, when the original color image having high SFM (baboon image).

Table 3. PSNRc of the denoised images generated from two denoising algorithms at noise variance 10,15 , and 20

\begin{tabular}{|c|c|c|c|c|c|c|}
\hline \multirow{2}{*}{ Image name } & \multicolumn{2}{|c|}{ noise variance $=10$} & \multicolumn{2}{c|}{ noise variance $=15$} & \multicolumn{2}{c|}{ noise variance $=20$} \\
\cline { 2 - 7 } & \multicolumn{2}{|c|}{ PSNRc } & \multicolumn{2}{c|}{ PSNRc } & \multicolumn{2}{c|}{ PSNRc } \\
\cline { 2 - 7 } & Proposed & Ref[17] & Proposed & Ref[17] & Proposed & Ref[17] \\
\hline pepper & 33.856 & 33.197 & 31.87 & 31.984 & 31.033 & 30.974 \\
\hline barbarra & 33.8688 & 34.0356 & 31.8873 & 31.7005 & 29.849 & 30.05 \\
\hline lenna & 33.6744 & 34.9019 & 33.1799 & 33.2943 & 32.1246 & 32.1289 \\
\hline baboon & 32.1835 & 29.1042 & 29.0568 & 27.5316 & 27.1416 & 26.2805 \\
\hline
\end{tabular}

Table 4. IFS of the denoised images generated from two denoising algorithms at noise variance 10,15 , and 20

\begin{tabular}{|c|c|c|c|c|c|c|}
\hline \multirow{2}{*}{ Image name } & \multicolumn{2}{|c|}{ Noise Variance $=10$} & \multicolumn{2}{c|}{ Noise Variance $=15$} & \multicolumn{2}{c|}{ Noise Variance $=20$} \\
\cline { 2 - 7 } & \multicolumn{2}{|c|}{ IFS } & \multicolumn{2}{c|}{ IFS } & \multicolumn{2}{c|}{ IFS } \\
\cline { 2 - 7 } & Proposed & Ref[17] & Proposed & Ref[17] & Proposed & Ref[17] \\
\hline Pepper & 0.9892 & 0.9844 & 0.979 & 0.977 & 0.971 & 0.97 \\
\hline Barbarra & 0.9868 & 0.9889 & 0.9809 & 0.9785 & 0.9684 & 0.9665 \\
\hline Lenna & 0.9856 & 0.9875 & 0.9797 & 0.9795 & 0.9710 & 0.9704 \\
\hline Baboon & 0.9935 & 0.9849 & 0.9856 & 0.9796 & 0.9768 & 0.9707 \\
\hline
\end{tabular}

For subjective assessment, the visual comparisons of the performance are shown in Figure 3 and 4 . From a visual perspective, the proposed noise estimation algorithm works comparatively well by giving more noise-free, less content distortion, and fewer noticeable color artifacts than using a robust median estimator on bivariate shrinkage functions, especially noticeable in the baboon nose and green pepper surface area. Note that, the execution of MATLAB implementation of our proposed method only lasts about 3 and 6 seconds for a color image size 256x256 and 512x512, respectively with Intel Core i5-4460T Processor $1.9 \mathrm{GHz}, 4 \mathrm{~GB}$ RAM. 


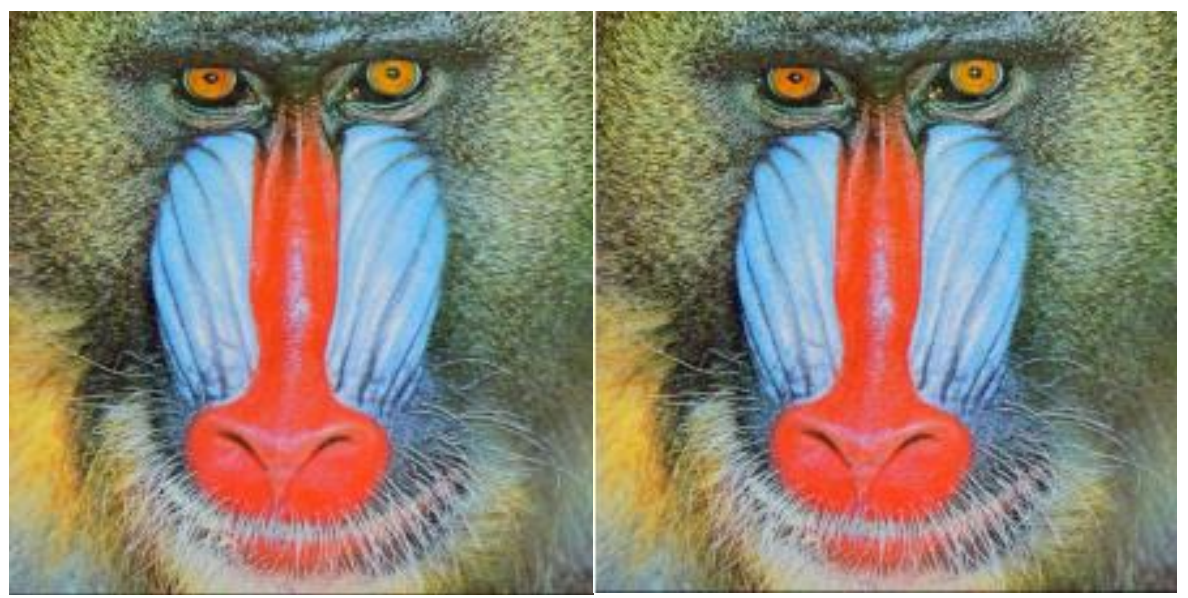

(a)

(b)

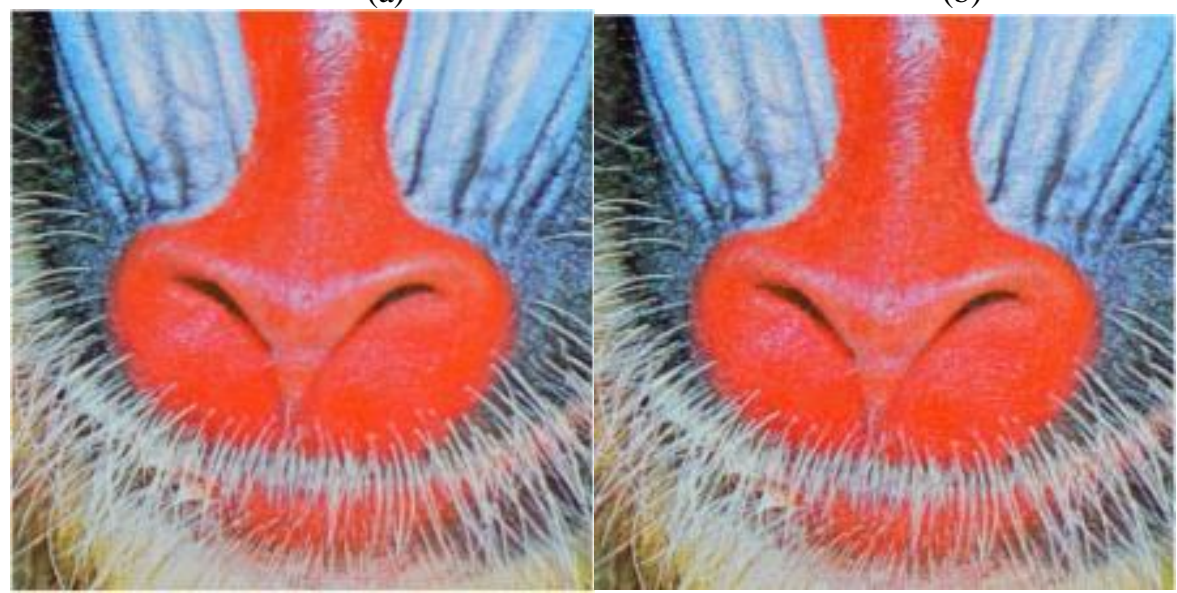

(c)

(d)

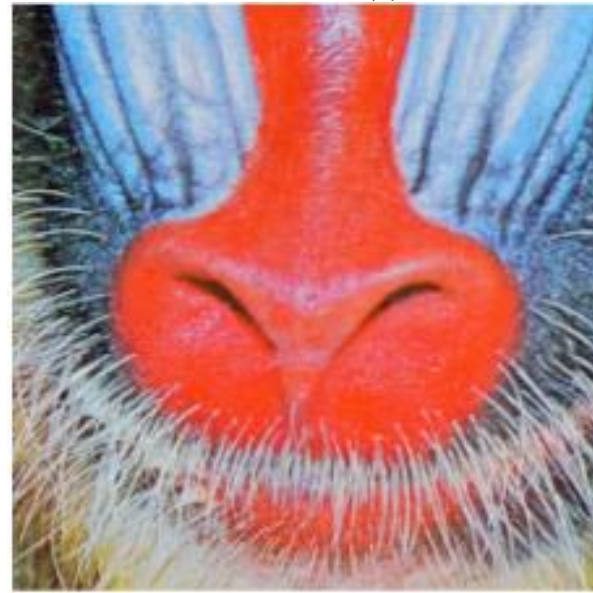

(e)

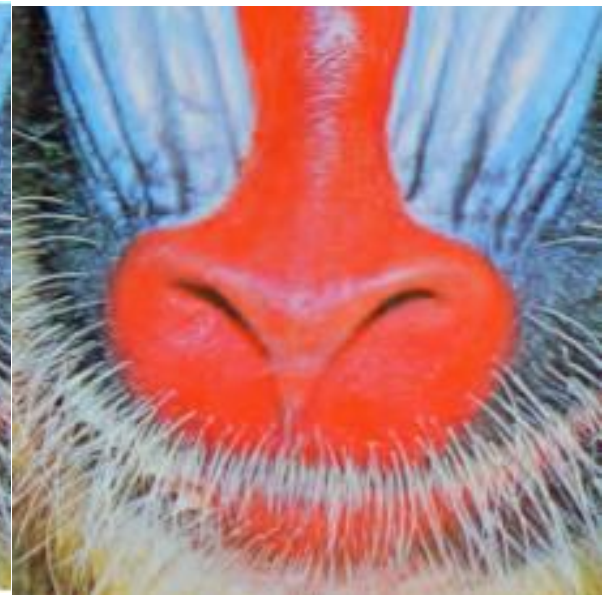

(f)

Figure 3. (a) Original color image of baboon (b) Noisy image with $\sigma=10$

(c) A cropped and zoom in of baboon image (d) A cropped and zoom in of noisy baboon image

(e) A cropped and zoom in of denoised image by proposed method

(f) A cropped and zoom in of denoised image by bivariate shrinkage functions method [17] 


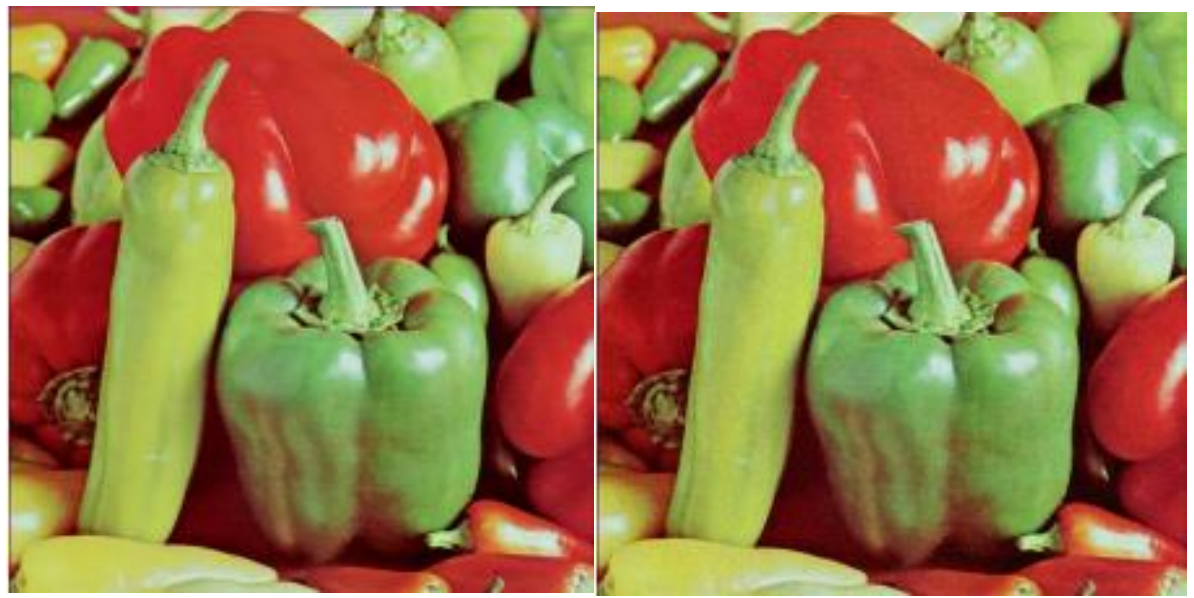

(a)

(b)

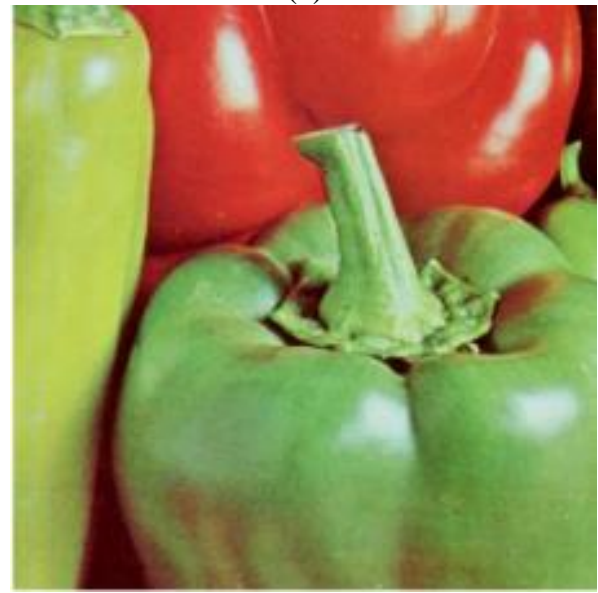

(c)

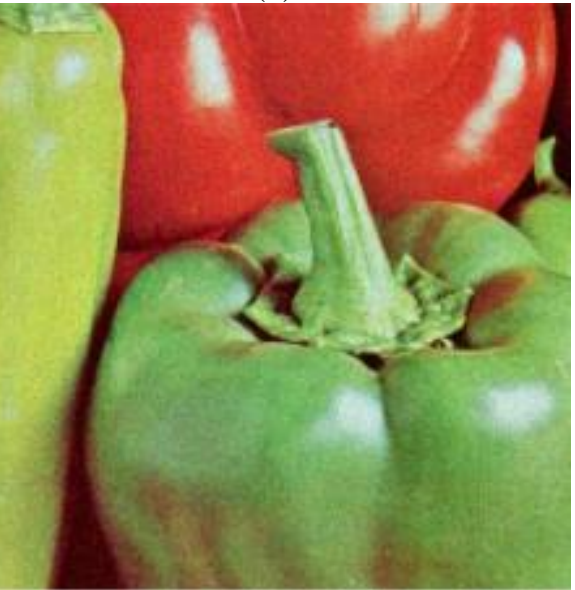

(d)

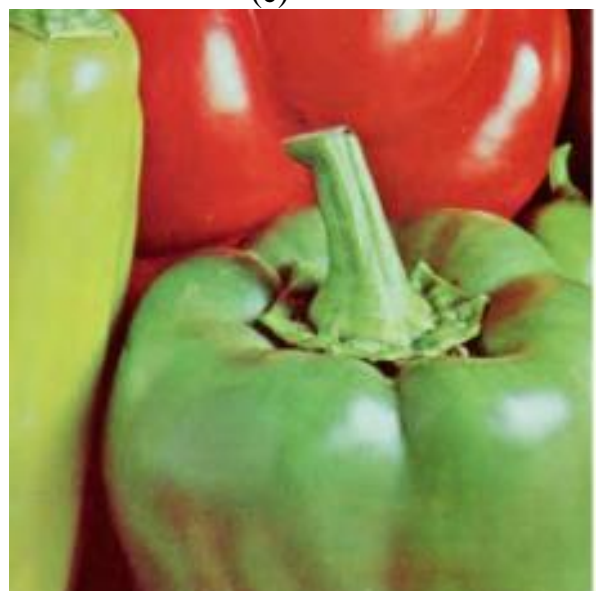

(e)

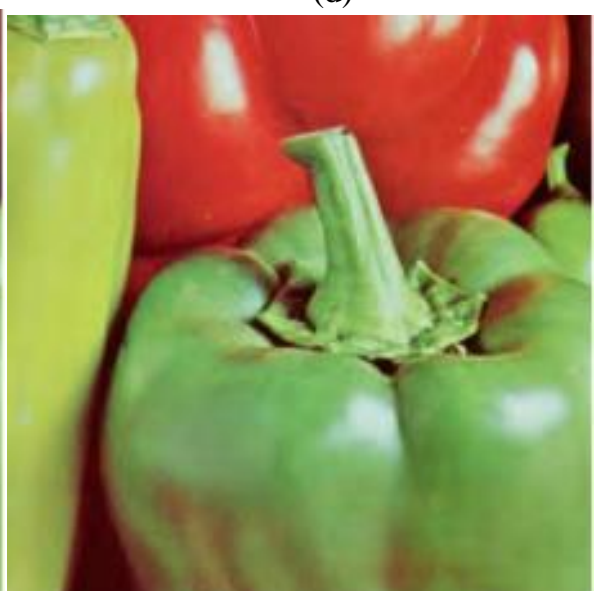

(f)

Figure 4. (a) Original color image of pepper (b) Noisy image with $\sigma=10$

(c) A cropped and zoom in of pepper image (d) A cropped and zoom in of noisy pepper image

(e) A cropped and zoom in of denoised image by proposed method

(f) A cropped and zoom in of denoised image by bivariate shrinkage functions method [17] 


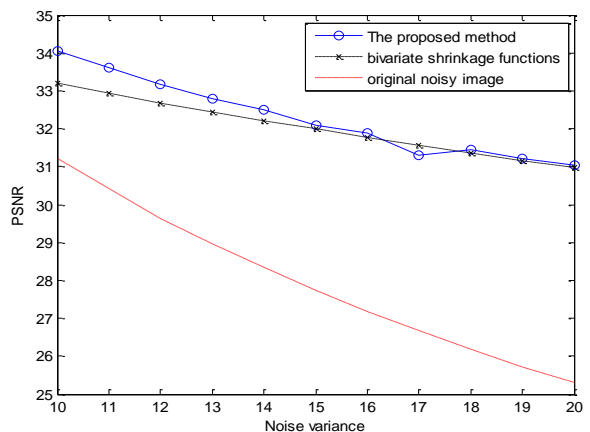

(a) Pepper image

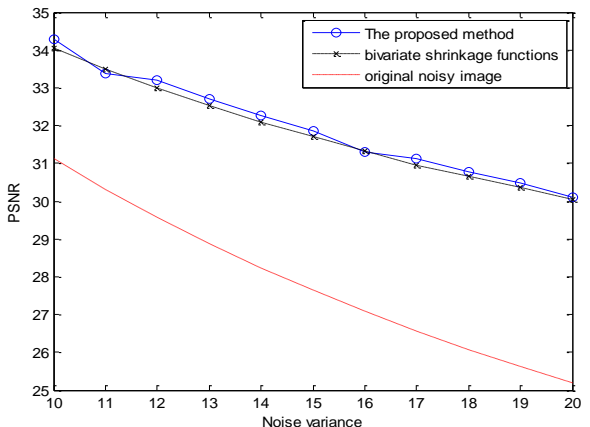

(b) Babara image

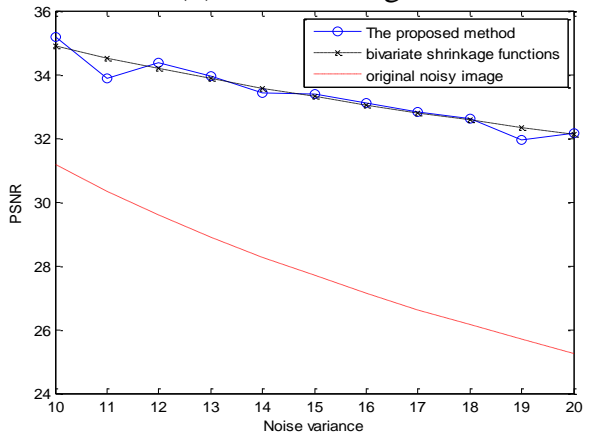

(c) Lenna image

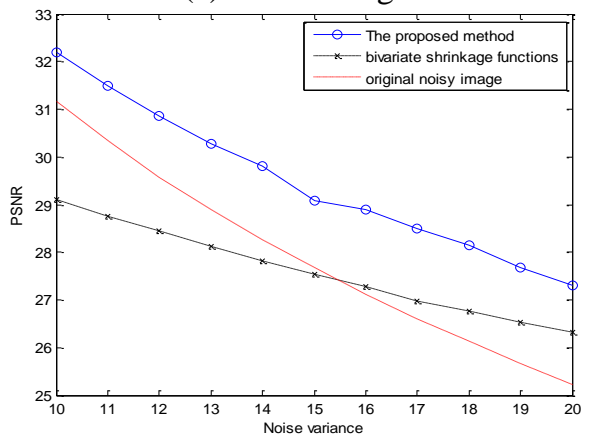

(d) Baboon image

Figure 5. Plotting of denoised image results using proposed method and bivariate shrinkage function 


\section{Conclusion and future work}

This research presents a noise estimation technique for multiple noisy color image copies. One application of this proposed technique is to be used in conjunction with other image noise reduction techniques to improve the quality of the recovered image in such scenario. Our proposed color image denoising technique had similar or slightly closed but performs better than well-known wavelet based denoising [17], bivariate shrinkage functions, when the original color image having high SFM. The large value of SFM means that image contain high frequency components. In addition, our proposed noise estimation is quite suitable for color noise reduction under low and high noise power conditions where noise can be minimized, the high frequency components of the image can be also preserved. However, this work only focuses on the simple case when only two noisy image copies are available. One area of the future work is to analyze the accuracy and confident level of the proposed estimation technique apply it to the application where more than two noisy image copies are available and where these images are not necessarily corrupted by the same noise variance. In addition, other color spaces need to be considered to improve our proposed method, for which we shall discuss details in our future work.

\section{Acknowledgment}

This work is supported by Rajamangala University of Technology Suvarnabhumi.

\section{References}

[1] F. Luisier and T. Blu, "SURE-LET multichannel image denoising: interscale orthonormal wavelet thresholding," IEEE Transactions on Image processing, vol. 17(4), pp. 482-492, 2008.

[2] A. Mcandrew, An Introduction to Digital Image Processing withMatlab, Thomson, 2004.

[3] D. L. Donoho, "De-noising by soft-thresholding," IEEE Transaction on Information Theory, vol. 41(3), pp. 613-627, 1995.

[4] S. G. Chang and Y. Bin, and M. Vetterli, "Adaptive wavelet thresholding for image denoising and compression," IEEE Transactions on Image Processing, vol. 9, pp. 15321546, 2000.

[5] H. Om and M. Biswas, "An adaptive image denoising method based on thresholding," WSEAS transactions on Signal Processing, vol. 10, pp. 1-8, 2014.

[6] S. Sutha, E. J. Leavline, D. A. Antony and G. Singh, "A comprehensive study on wavelet based shrinkage methods fordenoising natural images," WSEAS transactions on Signal Processing, vol. 9, pp. 203-215, Oct 2013.

[7] A. Jaiswal, J. Upadhyay and A. Somkuwar, "Image denoising and quality measurements by using filtering and wavelet based techniques," International Journal of Electronics and Communications, vol. 68, pp. 699-705, 2014.

[8] J. Scharcanski, C. R. Jung and R. T. Clarke, "Adaptive image denoisingdenoising using scale and space consistency," IEEE Transactions on Image Processing, vol. 6 (9), pp.10921101, 2002.

[9] S. Suhaila, R. Hazli, and T. Shimamura, "Smooth region's mean deviation-baseddenoising method," International Journal of Circuits, Systems and Signal Processing, vol. 7(3), pp. 191-198, 2013.

[10] S. Suhaila, and T. Shimamura, "Image restoration based on edgemap andwiener filter for preserving fine details andedges," International Journal of Circuits, Systems and Signal Processing, vol.5(6), pp. 618-626, 2011.

[11] J. Dai, O. C. Au, L. Fang, C. Pang, F. Zou, and J. Li, "Multichannel nonlocal means fusion forcolor image denoising," IEEE Transactions on circuits and systems for video technology, vol.23(11), pp. 1873-1886, Nov 2013.

[12] S. Meher, "Color Image Denoising withMulti-channel Circular Spatial Filtering," IEEE Int. Conf. onComputer Modelling and Simulation Communications,pp. 284-288, 2010.

[13] N. X. Lian, V. Zagorodnov, and Y. P. Tan, "Color image denoising using wavelets and minimum cut analysis,” IEEE Signal processing letters, vol. 12 (11), pp.741-744, Nov 2015. 
[14] S. G. Chang, B. Yu, and M. Vetterli, "Wavelet thresholding for multiple noisy image copies", IEEE Transactions on Image Processing, vol. 9 (9), pp. 1631-1635, 2000.

[15] K. Youssef, N. Jarenwattananon, and L. Bouchard, "Feature-preserving noise removal", IEEE Transactions on Medical Imaging, vol. 34, pp. 1822-1829, 2015.

[16] S. Udomhunsakul and N. Sae-bae, "Noise estimation techniquefor multiple copies image denoising," Int. Conf. on Intelligent systems and Image processing, 2016, pp. 105-110.

[17] L. Sendur and I. W. Selesnick, "Bivariate shrinkage functions for wavelet-based denoising exploiting interscale dependency”, IEEE Transactions on Signal Processing,vol. 50(11), pp. 2744-2756, 2002.

[18] Matlab implementation of wavelet transforms [online].Available:http://eeweb.poly.edu/iselesni/WaveletSoftware/index.html.

[19] K. Q. Huang, Q. Wang and Z. Yang. Wu, "Natural color image enhancement and evaluation algorithm based on human visual system," Computer Vision and Image Understanding, vol. 103, pp. 52-63, 2006.

[20] S. Grgic, M. Grgic, and M. Mrak, "Reliability of Objective Picture Quality Measurement," Journal of Electrical Engineering, vol. 55(1-2), pp. 3-10, 2004.

[21] Z. Wang, A. Bovik, H. Sheikh, and E. Simoncelli, "Image Quality Assessment: From Error Visibility to Structural Similarity," IEEE Transactions on Image processing, Vol.13 (4), pp. 600-612, 2004.

[22] R. Moreno, M. A. Garcia, D. Puig and C. Julia, "Edge-preserving color image denoising through tensor voting," Computer Vision and Image Understanding, vol. 115, pp. 15361551, 2011.

[23] H. W. Chang, Q. W. Zhang, Q. G. Wu and Y. Gan, "Perceptual image quality assessment by independent feature detector," Neurocomputing, Vol.151, pp. 1142-1152, 2015.

[24] Perceptual image quality assessment by independent feature detector [online]. Available:https://www.mathworks.com/matlabcentral/fileexchange/49558-perceptualimage-quality-assessment-by-independent-feature-detector.

[25] The usc-sipi image database [online]. Available: http://sipi.usc.edu/database/database.php.

[26] Set of classic test images. [online]. Available:http://www.hlevkin.com/TestImages.

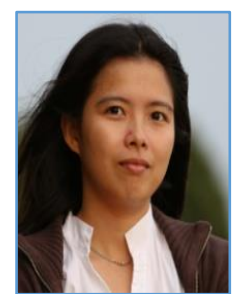

Napa Sae-Bae received her $\mathrm{PhD}$ in Computer Science from Polytechnic School of Engineering, New York University in 2014, whose advisors are Prof. Nasir Memon and Prof. Katherine Isbister. She currently holds a faculty position at computer science department, faculty of science and technology, Rajamangala University of Technology Suvarnabhumi. Her research interests lie in the area of biometric, authentication, consumer security, pattern recognition, signal processing, and image processing, e-mail: benapa@ gmail.com.

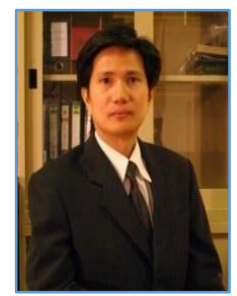

Somkait Udomhunsakul received his doctoral degree in Electrical Engineering from Florida Institute of Technology in 2003, whose advisor is Prof. Dr. Samuel Kozaitis. He then worked as an assistant professor at Information Engineering Department, King Mongkut's Institute of Technology Ladkrabang from June 2003 to October 2010. He is currently an associate professor at Rajamangala University of Technology Suvarnabhumi, Faculty of Engineering and Architecture, Telecommunication and Electronic Engineering Department. His research interest is digital image processing, email:sudomhun@hotmail.com. 